\title{
Correction to: International prospective observational cohort study of Zika in infants and pregnancy (ZIP study): study protocol
}

\author{
Jill F. Lebov ${ }^{1 *}$, Juan F. Arias ${ }^{2}$, Angel Balmaseda ${ }^{3}$, William Britt ${ }^{4}$, José F. Cordero ${ }^{5}$, Luiz Augusto Galvão ${ }^{6}$, \\ Ana Lucía Garces ${ }^{7}$, K. Michael Hambidge ${ }^{8}$, Eva Harris ${ }^{9}$, Albert Ko ${ }^{10,11}$, Nancy Krebs ${ }^{8}$, Ernesto T. A. Marques ${ }^{12,13}$, \\ Alexander M. Martinez ${ }^{14}$, Elizabeth McClure ${ }^{1}$, Democrito B. Miranda-Filho ${ }^{15}$, Maria Elisabeth Lopes Moreira ${ }^{16}$, \\ Marisa M. Mussi-Pinhata ${ }^{17}$, Theresa J. Ochoa ${ }^{18}$, Jorge E. Osorio ${ }^{19}$, Deolinda M. F. Scalabrin ${ }^{10,11}$, \\ Stacey Schultz-Cherry ${ }^{2}$, George R. Seage $\|^{20}{ }^{2}$, Kristen Stolka ${ }^{1}$, César Augusto Ugarte-Gil ${ }^{18}$, \\ Carmen Milagros Velez Vega ${ }^{21}$, Michael Welton ${ }^{5}$, Ricardo Ximenes ${ }^{22}$ and Carmen Zorrilla ${ }^{23}$
}

\section{Correction to: BMC Pregnancy Childbirth https://doi.org/10.1186/s12884-019-2430-4}

Following publication of the original article [1], the author mentioned that two additional NIH staff were involved in the development of the protocol who did not receive recognition in the Acknowledgments section in their published article. He would like to add the following people to the Acknowledgements section:

\section{Martha Nason and Erin Gabriel}

The publisher apologizes for any inconvenience caused by this error.

\footnotetext{
Author details

'Social, Statistical and Environmental Sciences, RTI International, Durham, NC, USA. ${ }^{2}$ Department of Infectious Diseases, St Jude Children's Research Hospital, Memphis, TN 38105, USA. ${ }^{3}$ Centro Nacional de Diagnostico y Referencia, Complejo Nacional de Salud, Managua, Nicaragua. ${ }^{4}$ Department of Pediatrics, University of Alabama at Birmingham, Birmingham, AL, USA. ${ }^{5}$ Department of Epidemiology and Biostatistics, College of Public Health, University of Georgia, Athens, GA, USA. ${ }^{6}$ Center for Global Health - CRIS, FIOCRUZ, Rio de Janeiro, Brazil. ${ }^{7}$ Fundación para la Alimentación y Nutrición de Centro América y Panamá (INCAP), Guatemala City, Guatemala. ${ }^{8}$ Section of Nutrition, Pediatrics, University of Colorado, Aurora, CO, USA. ${ }^{9}$ Division of Infectious Diseases and Vaccinology, School of Public Health, University of California, Berkeley, CA, USA. ${ }^{10}$ Department of Epidemiology of Microbial
}

Diseases, Yale School of Public Health, New Haven, CT, USA. ${ }^{11}$ Instituto Gonçalo Moniz, Fundação Oswaldo Cruz/MS, Salvador, Brazil. ${ }^{12}$ School of Public Health, University of Pittsburgh, Pittsburgh, PA, USA. ${ }^{13}$ Instituto Aggeu Magalhães, Department of Virology and Experimental Therapeutics, FIOCRUZ, Pernambuco, Brazil. ${ }^{14}$ Director of Research Institute at Imbanaco Medical Center, Cali, Colombia. ${ }^{15}$ Programa de Pós-Graduação em Ciências da Saúde (PPGCS) da Universidade de Pernambuco, Microcephaly Epidemic Research Group, Recife, Brazil. ${ }^{16}$ Instituto Fernandes Figueira - FIOCRUZ, Rio de Janeiro, Brazil. ${ }^{17}$ Ribeirão Preto Medical School, Ribeirão Preto, Brazil.

${ }^{18}$ Instituto de Medicina Tropical Alexander von Humboldt and Facultad de Medicina, Universidad Peruana Cayetano Heredia, Lima, Peru. ${ }^{19}$ Department of Pathobiological Sciences, University of Wisconsin, Madison, WI, USA. ${ }^{20}$ Department of Epidemiology, Harvard Chan School of Public Health, Boston, MA, USA. ${ }^{21}$ University of Puerto Rico, San Juan, Puerto Rico. ${ }^{22}$ Departamento de Medicina Tropical da Universidade Federal de Pernambuco, Microcephaly Epidemic Research Group, Recife, Brazil.

${ }^{23}$ Maternal-Infant Studies Center (CEMI), San Juan, Puerto Rico.

Published online: 19 November 2019

Reference

1. Lebov, et al. International prospective observational cohort study of Zika in infants and pregnancy (ZIP study): study protocol. BMC Pregnancy Childbirth. 2019;19:282. https://doi.org/10.1186/s12884-019-2430-4.

The original article can be found online at https://doi.org/10.1186/s12884019-2430-4

* Correspondence: jlebov@rti.org

${ }^{1}$ Social, Statistical and Environmental Sciences, RTI International, Durham, NC, USA

Full list of author information is available at the end of the article

(c) The Author(s). 2019 Open Access This article is distributed under the terms of the Creative Commons Attribution 4.0 International License (http://creativecommons.org/licenses/by/4.0/), which permits unrestricted use, distribution, and reproduction in any medium, provided you give appropriate credit to the original author(s) and the source, provide a link to the Creative Commons license, and indicate if changes were made. The Creative Commons Public Domain Dedication waiver (http://creativecommons.org/publicdomain/zero/1.0/) applies to the data made available in this article, unless otherwise stated. 